\title{
Evaluation of Network Stack Optimization Techniques for Wireless Sensor Networks
}

\author{
Jaein JEONG \\ Computer Science Division, UC Berkeley, Berkeley, California, USA \\ Email:jaein@eecs.berkeley.edu \\ Received August 19, 2009; revised September 20, 2009; accepted October 10, 2009
}

\begin{abstract}
We present a network stack implementation for a wireless sensor platform based on a byte-level radio. The network stack provides error-correction code, multi-channel capability and reliable communication for a high packet reception rate as well as a basic packet-level communication interface. In outdoor tests, the packet reception rate is close to $100 \%$ within $800 \mathrm{ft}$ and is reasonably good up to $1100 \mathrm{ft}$. This is made possible by using error correction code and a reliable transport layer. Our implementation also allows us to choose a frequency among multiple channels. By using multiple frequencies as well as a reliable transport layer, we can achieve a high packet reception rate by paying additional retransmission time when collisions increase with additional sensor nodes.
\end{abstract}

Keywords: Wireless Sensors, Network Stack, Error Correction Code, Reliable Transport, Multi Channel

\section{Introduction}

In wireless sensor networks, it is desirable to have sensor nodes communicate without packet loss allowing information from a sensor node to be transferred reliably. In reality, however, sensor nodes suffer different levels of packet loss due to signal attenuation and multi-path effect [1-3]. In this paper, we take a practical approach to address this problem. We implement a network stack as an experiment vehicle based on a byte-level radio, the CC1000 transceiver [4]. Besides the basic packet-level communication, the network stack provides additional functionality for performance improvement such as error-correction code, retransmission and channel diversity. Based on the network stack we have implemented, we have evaluated how this extended functionality improves communication performance. Our network stack has reasonable performance in an outdoor environment with the packet reception rate close to $100 \%$ within an $800 \mathrm{ft}$ range. We have found that error-correction code, retransmission and channel diversity improves this performance even further. For error-correction code, we have used SECDED (Single-Error Correction and Double-Error Detection) code [5,6]. Using SECDED code improved the packet reception rate, but it was not effective when packets were completely lost due to the multipath effect. Retransmission was effective in reducing packet losses from the multi-path effect and contention from traffic. We also saw that using multiple radio channels was very effective in reducing collisions when multiple senders burst packets.

The rest of this paper is organized in the following way: Section 2 overviews background and related work; Section 3 describes the design principles of our network stack; Section 4 empirically evaluates our network stack and Section 5 concludes this paper.

\section{Background and Related Work}

TinyOS provides communication capability using multiple layers like other networked systems: application, transport, network, and link layer. An application sees the radio as a service interface through which it can send and receive data in fixed sized packets. The transport layer provides best effort delivery and process-to-process communication. The network layer provides a routing tree. The link layer converts a packet to and from the byte data and interacts with the underlying radio chip. Since the link layer is so closely coupled with the radio chip, writing a network stack for a new platform usually requires rewriting the link layer.

Our project is based on some earlier works. Hill wrote a preliminary version of a network stack for the $\mathrm{CC} 1000$ 
radio supporting Active Message number multiplexing and packet framing [7]. The CC1000 can operate in one of three frequency bands $(433 \mathrm{MHz}, 866 \mathrm{MHz}$ and 900 $\mathrm{MHz}$ ) and each band requires different values for external components and initialization parameters. The choice of frequency band is determined by the coverage and the number of legally usable channels: $900 \mathrm{MHz}$ is preferred for its relatively large selection of channels and $433 \mathrm{MHz}$ for its longer range. Hill initially wrote a network stack for the CC1000 radio in the $900 \mathrm{MHz}$ range and Crossbow technology modified it to support the $433 \mathrm{MHz}$ range. From the earlier work done by Hill and Crossbow technology, we found some room for improvement: 1) supporting error correction code to protect data from transient errors, 2) utilizing multiple channels of the CC1000 radio chip, 3) supporting reliable communication.

\section{Design}

In this section, we describe mechanism and design philosophy of our network stack for the Mica2dot platform [8] that is based on a byte-level radio transceiver CC1000 radio chip and the ATMega103L microcontroller [9], illustrated in Figure 1. First, we describe how we provide byte-level data and configuration interfaces (SpiByteFifoC and ChipconC modules in Figure 1) to

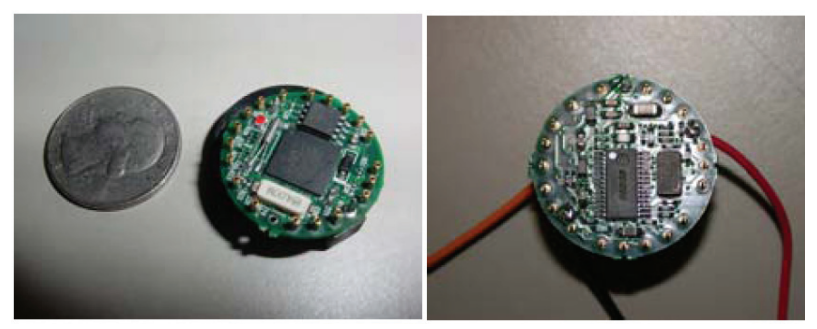

(a) Mica2dot node - front and back

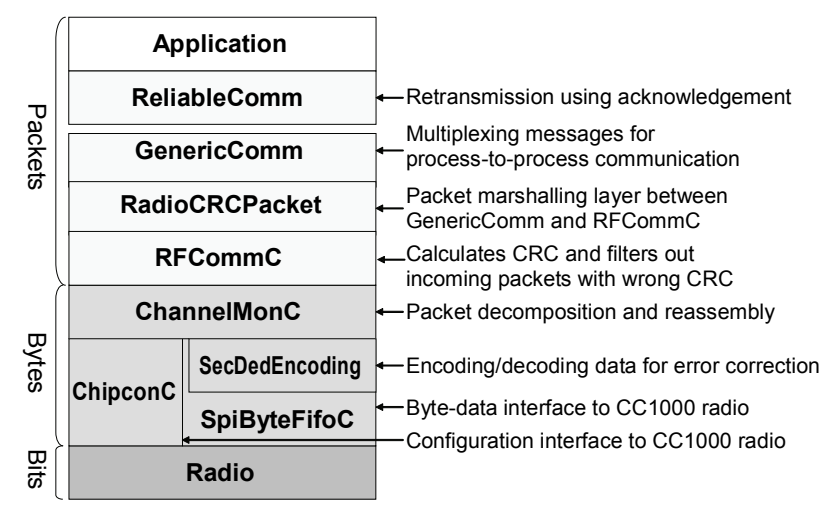

(b) Mica2dot network stack and description of its layers

Figure 1. Mica2dot and its network stack. abstract the low-level interface of the $\mathrm{CC} 1000$ radio with generic byte-level interfaces. Second, we describe how we provide a packet-level interface for the $\mathrm{CC} 1000$ radio (ChannelMonC module). Third, we describe how we provide error-correction capability to protect data from transient errors (SecDedEncoding module). Fourth, we describe how we provide an interface to utilize the diversity of multiple radio channels (ChipconC module). Fifth, we describe how we support reliable communication using retransmission (ReliableComm module).

\subsection{Abstracting Radio Hardware}

1) Byte-level data interface: While at the physical level, the ATMega103L microcontroller communicates with the $\mathrm{CC} 1000$ radio in bits over two serial pins, it provides a byte-level interface through the SPI (Serial Peripheral Interface) registers: SPSR, SPDR and SPCR.

- SPSR (SPI Status Register) can be used to check whether there is an incoming byte or not. The most significant bit (bit 7) of the SPSR becomes high when there is an incoming byte in the buffer, low otherwise. The CC1000 radio can be switched between send and receive mode by changing the data direction of the data pin (bit-3 of port B of ATMega103L microcontroller).

- SPDR (SPI Data Register) is a byte buffer, which assembles either outgoing or incoming bits into a byte before reading or writing data to and from the $\mathrm{CC} 1000$ radio. When incoming bits are assembled into a byte, the ATMega103L microcontroller triggers SIG_SPI interrupt as well as setting bit 7 of the SPSR. This allows incoming data for the $\mathrm{CC} 1000$ to be efficiently processed using an interrupt instead of polling. One note is that only a send or a receive can be done at any time because the CC1000 radio has only a single buffer.

- SPCR (SPI Control Register) can be used to enable or disable interrupts. The SPI clock interrupt is triggered when SPCR is set to 0xc0, and is disabled when set to 0x40. Finally, data should be read or written at the same rate with that of the external device. The $\mathrm{CC} 1000$ radio is synchronized to the ATMega103L microcontroller through the SPI clock. The clock triggers an output pin of the microcontroller (bit-1 of port B) to the data clock pin of the radio chip (SPI CLK).

HPLSpiC and SpiByteFifoC are TinyOS modules that abstract the data interface from the ATMega103L microcontroller to the $\mathrm{CC} 1000$ radio. The methods for these modules are summarized in Figure 2(a) and Table 1.

2) Serial configuration interface: While the CC1000 radio can transmit or receive data through the data interface, it needs to be configured for initialization or property changes such as radio frequency (explained in the next subsection) or transmission power level. The CC1000 radio exposes three pins PALE, PCLK and 


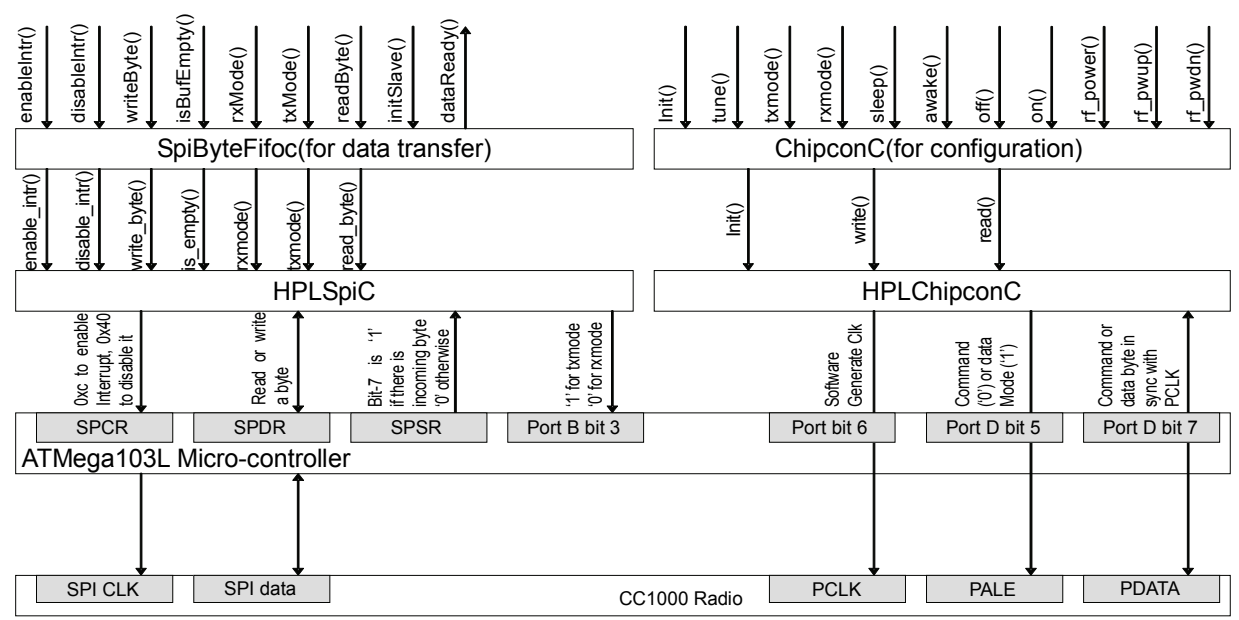

(a) Hardware abstraction for $\mathrm{CC} 1000$ radio - SpiByteFifoC and ChipconC modules provide higher-level abstraction over CC1000 radio hardware

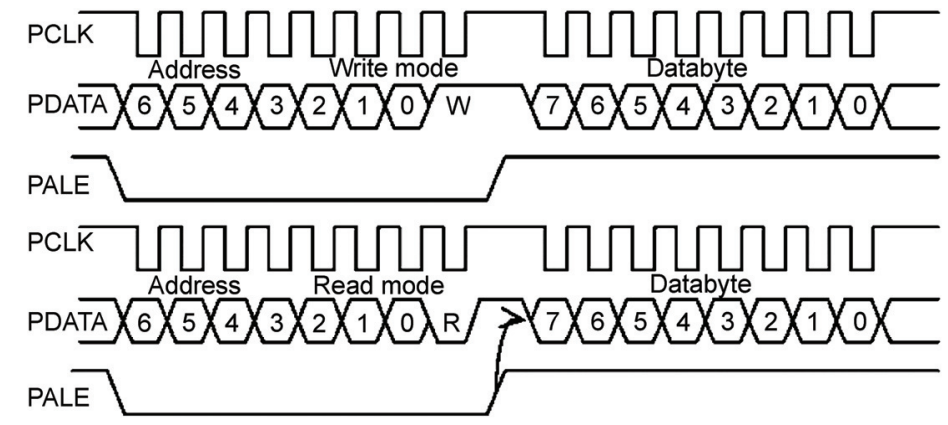

(b) Bit sequence for accessing CC1000 control registers - i) write a byte, ii) read a byte
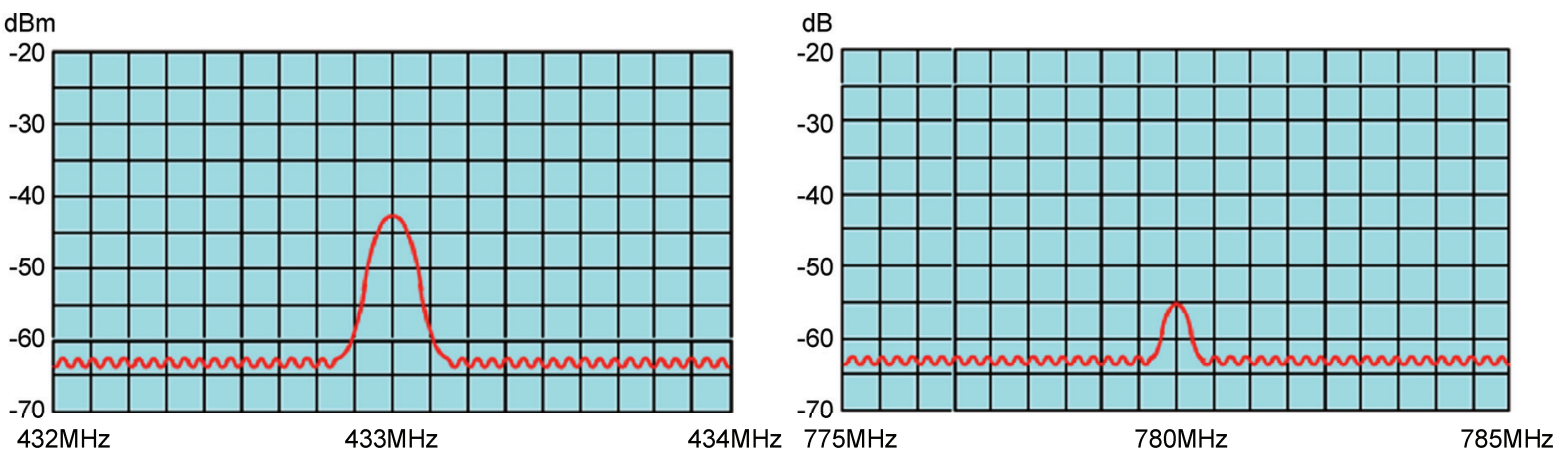

(c) Waveform of CC1000 in spectrum analyzer - i) correctly configured, ii) misconfigured. Notice that the peak is over 10dB higher when the radio is correctly configured compared to when it is misconfigured.

Figure 2. Abstracting radio hardware.

PDATA for this purpose as shown in Figure 2(a). These pins are mapped to data port pins (port D bit 5, 6 and 7). By setting or clearing these pins, the microcontroller can send a sequence of bits (control register address, a byte of data and a bit representing whether the operation is write or read as it is shown in Figure 2(b). This is implemented as init () , write () and $\operatorname{read}()$ in the HPLChipconC module (Table 2). The ChipconM module implements high level functions such as setting the radio frequency or transmission power using these primitives.
3) Using multiple channels: The CC1000 radio allows us to select a channel at run time among a number of frequencies. The purpose of selecting channels is to reduce any interference from neighboring sensor nodes or other wireless devices. For the CC1000 radio chip to operate at a specific frequency, it needs to be configured with the correct frequency words and clock divisor byte. CC1000 transmits and receives at different frequencies and these frequencies are represented by two 24-bit frequency words. These frequencies are generated by dividing the 
Table 1. Byte-level data interface to the $\mathrm{CC1000}$ radio.

\begin{tabular}{ll} 
HPLSpiC & \multicolumn{1}{c}{ Method } \\
\hline HPLSpi. enable_intr() & Enables SPI clock interrupt (sets SPCR to 0xC0) \\
HPLSpi. disable_intr() & Disables SPI clock interrupt (sets SPCR to 0x40) \\
HPLSpi. write_byte() & Writes a byte (writes a byte to SPDR) \\
HPLSpi. read_byte() & Reads a byte (reads a byte from SPDR) \\
HPLSpi. is_empty() & Bit-7 is '1' if there is an incoming byte, '0' otherwise \\
HPLSpi. txmode() & Switches to transmit mode (sets bit-3 of PortB as '1') \\
HPLSpi. rxmote() & Switches to transmit mode (sets bit-3 of PortB as '0') \\
\hline SpiByteFifoC & \multicolumn{1}{c}{ Method } \\
\hline SpiByteFifo. initSlave() & Initializes the SPI registers \\
SpiByteFifo. dataReady() & Called when there is an incoming byte \\
SpiByteFifo. enableIntr() & Calls HPLSpi.enable_intr( ) \\
SpiByteFifo. diableIntr() & Calls HPLSpi.diable_intr( ) \\
SpiByteFifo. writeByte() & Calls HPLSpi.write_byte( ) \\
SpiByteFifo. readByte() & Calls HPLSpi.read_byte( ) \\
SpiByteFifo. isBufEmpty() & Calls HPLSpi.is_empty( ) \\
SpiByteFifo. txMode() & Calls HPLSpi.txmode( ) \\
SpiByteFifo. rxMode() & Calls HPLSpi.rxmode( ) \\
\hline &
\end{tabular}

Table 2. Serial configuration interface to the $\mathrm{C} 1000$ radio.

HPLChipconC

\begin{tabular}{|c|c|}
\hline Method & Description \\
\hline HPLChipcon.init() & Initializes $\mathrm{CC} 1000$ configuration registers \\
\hline HPLChipcon.write() & Writes a byte to the $\mathrm{CC} 1000$ register with the given 7-bit address \\
\hline HPLChipcon.read() & Reads a byte from the $\mathrm{CC} 1000$ register with the given 7-bit address \\
\hline \multicolumn{2}{|l|}{ ChipconM } \\
\hline Method & Description \\
\hline Chipcon. init() & Initializes CC1000 configuration registers and tunes the radio frequency to the given value \\
\hline Chipcon. tune() & Tunes the radio frequency to the given value \\
\hline Chipcon. txmode() & Sets the CC1000 in transmit mode \\
\hline Chipcon. rxmode() & Sets the CC1000 in receive mode \\
\hline Chipcon. sleep () & Puts the CC1000 in the sleep mode \\
\hline Chipcon. awake() & Awakes the CC1000 radio from the sleep mode \\
\hline Chipcon. off() & Turns off the CC1000 radio \\
\hline Chipcon. on() & Turns on the $\mathrm{CC} 1000$ radio \\
\hline Chipcon.rf_power() & Sets the transmit power for the $\mathrm{CC} 1000$ radio \\
\hline Chipcon.rf_pwup() & Increments the transmit power for the $\mathrm{CC} 1000$ radio \\
\hline Chipcon.rf_pwdn() & Decrements the transmit power for the $\mathrm{CC} 1000$ radio \\
\hline
\end{tabular}


Table 3. Channels available for Mica2dot in 433MHz band.

\begin{tabular}{lcccc}
\hline & CH1 & CH2 & CH3 & CH4 \\
\hline Parameter for Chipcon.init() or Chipcon.tune( ) & 0 & 1 & 2 & 3 \\
Tx freq (MHz) & 433.02 & 433.64 & 434.20 & 434.71 \\
Reg4-6 & $57 f 785$ & 581785 & 583785 & 585785 \\
Rx freq (MHz) & 433.09 & 433.71 & 434.27 & 434.78 \\
Reg4-6 & 580000 & 582000 & 584000 & 586000 \\
Reg12 divisor (PLL) & 60 & 60 & 60 & 60 \\
Output power (dBm) & -45 & -45 & -47 & -47 \\
\hline
\end{tabular}

frequency synthesizing clock (we are using 14.7456 $\mathrm{MHz}$ ) with the clock divisor byte. These values are set up in the ChipconM module.

The $\mathrm{CC} 1000$ radio can operate on one of the three frequency range $433 \mathrm{MHz}, 868 \mathrm{MHz}$ or $900 \mathrm{MHz}$, depending on the selection capacitor and inductor values for the resonator and the filter in the assembled hardware. While $900 \mathrm{MHz}$ is preferable for the wider selection of frequencies, we chose the $433 \mathrm{MHz}$ band in favor of longer range. Recommended values are listed in [4], but none of them worked for the $433 \mathrm{MHz}$ band. We found four working channels by measuring signal strength for different values between $433 \mathrm{MHz}$ and $435 \mathrm{MHz}$, as shown in Table 3.

Figure 2(c) shows the waveforms from a spectrum analyzer when the configuration is correct and wrong. In Figure 2(c)-(i), all the external components such as the resonator and filter are set to $433 \mathrm{MHz}$ band and the control register of $\mathrm{CC} 1000$ is correctly configured. The waveform has a peak at around $433 \mathrm{MHz}$ and its peak output power had around $-45 \mathrm{dBm}$ using an inducting antenna in the spectrum analyzer input. In Figure 2(c)-(ii), control registers are set to $433 \mathrm{MHz}$, but the inductor in the resonator is set to the value used in the $900 \mathrm{MHz}$ band. Since this resonator value doesn't match the other external components and the configuration value, its results at its peak is somewhere in the middle between $433 \mathrm{MHz}$ and $900 \mathrm{MHz}$ and its output power is much weaker than it should be. Actually, the initial build of the 433MHz Mica2dot nodes had this bug, and had to be addressed in the early stages of our project.

\subsection{Providing Packet-Level Interface}

Packet decomposition and reassembly is done in ChannelMonC with the help of the SpiByteFifoC module. SPI is synchronized to the microcontroller by the clock and generates an interrupt at regular intervals. At each interrupt invocation, the interrupt handler SIG_SPI in the SpiByteFifoC module is called. Then, we can determine if we can send or receive a byte by looking at the control register (SPSR) as it is shown in Figure 3(a).

Initially, it is in the IDLE state. If no incoming bytes are available, ChannelMonC sends a packet. Since the radio chip transfers data in bytes, we need to signal the beginning and the end of a packet. This is done by having a special sequence of bytes (preamble and start symbol) at the beginning and a fixed number of bytes after that. After sending the preamble and start symbol, ChannelMonC sends the data bytes. Data bytes can be

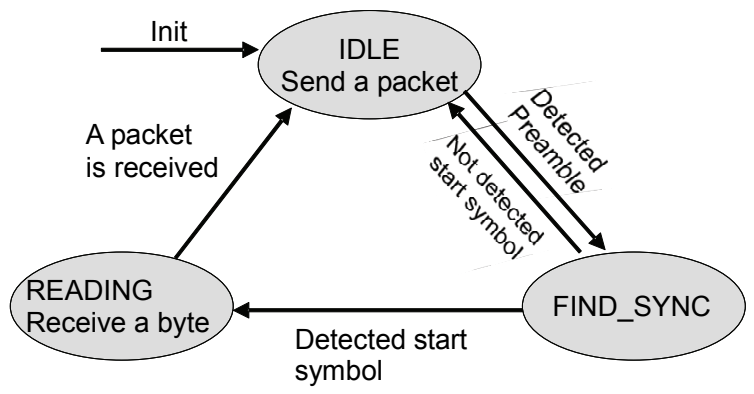

(a) State transition diagram for packet decomposition and reassembly

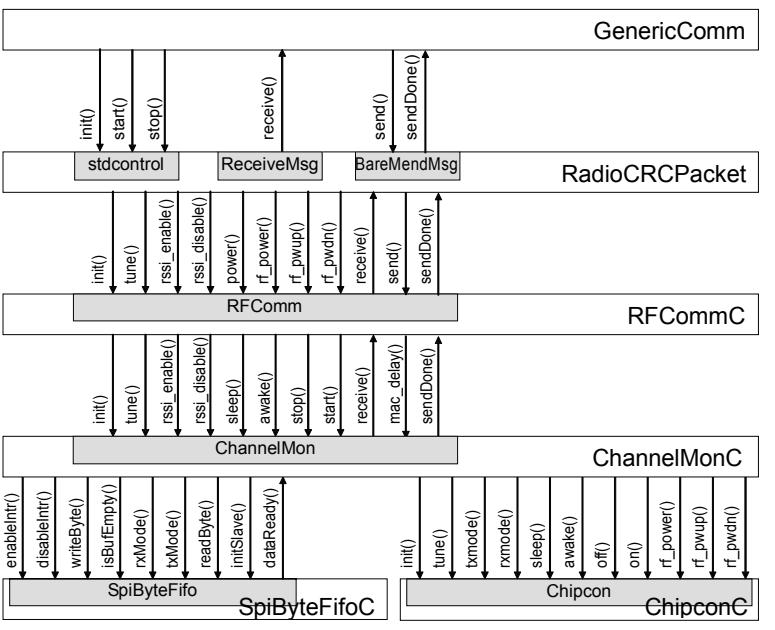

(b) Packet level interface in the network stack

Figure 3. Providing packet-level interface. 
sent as they are or can be sent after being encoded with error correction code for integrity. We used the SecDedEncoding module which implements a single-errorcorrection-and-double-error-detection (SECDEC) code. The version of ChannelMonC with error correction code is ChannelMonEccC. When there is an incoming byte, ChannelMonC reads the byte and sees whether the sequence of bytes received matches the preamble. If it does match, it goes to the FIND_SYNC state. If the next incoming bytes match the start symbol, it goes to the READING state. After reading the fixed length of data ( 36 bytes is the default), ChannelMonC notifies the arrival of a packet to the RFCommM module. The packet interface in the network is summarized in Figure 3(b).

\subsection{Providing Error-Correction Code}

In this section, first, we describe a coding theory based on linear block codes over field GF(2), which can be implemented in a simple and efficient way on resource constrained wireless sensor nodes. Then, we describe our implementation of error correction code based on linear block codes. The general process of encoding, transmis- sion and decoding of the message is shown in Figure 4(a).

1) Theory: The encoding of message $\mathbf{u}$ into codeword $\mathbf{v}$ can be achieved by multiplying the message $\mathbf{u}$ with the generation matrix $\mathbf{G}$. For data of width $k$-bits, $\mathbf{G}$ is of the form $\left[\mathbf{I}_{k}: \mathbf{C}\right]$, where $\mathbf{I}_{k}$ is the $k$-by- $k$ identity matrix and $\mathbf{C}$ is the $k$-by- $r$ binary matrix.

On the receiver end, a syndrome is calculated for detecting and possibly correcting errors. The syndrome $\mathbf{s}$ is calculated from the received signal $\mathbf{r}$ and the parity matrix $\mathbf{H}$. The parity matrix $\mathbf{H}$ is constructed from the generator matrix $\mathbf{G}$ and is of the form $\mathbf{H}=\left[\mathbf{C}^{T}: \mathbf{I}_{r}\right]$, where $\mathbf{r}$ is the number of parity bits. Denoting the error vector by e, we have

$$
\mathbf{s}=\mathbf{r} \mathbf{H}^{\mathrm{T}}=(\mathbf{v}+\mathbf{e}) \mathbf{H}^{\mathrm{T}}=\mathbf{u} \mathbf{G H}^{\mathrm{T}}+\mathbf{e H}^{\mathrm{T}}=\mathbf{e H}^{\mathrm{T}}
$$

Here, $\mathbf{G H}^{T}=\left[\mathbf{I}_{k}: \mathbf{C}\right]\left[\mathbf{C}^{T}: \mathbf{I}_{r}\right]^{T}=\mathbf{C}+\mathbf{C}=\mathbf{0}$ (addition is in $\mathrm{GF}(2)$ ). The non-zero $\mathbf{s}$ implies that an error occurred. Depending on the capability of the error correction code, the non-zero syndrome is compared with a row or a sum of rows of $\mathbf{H}^{T}$. If there are such rows, the correct codeword can be determined by flipping corresponding bits in the received signal.

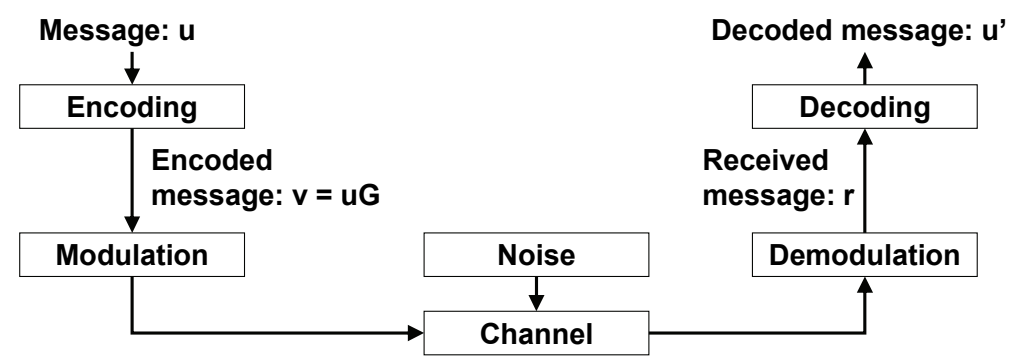

(a) Encoding, transmission and decoding of message

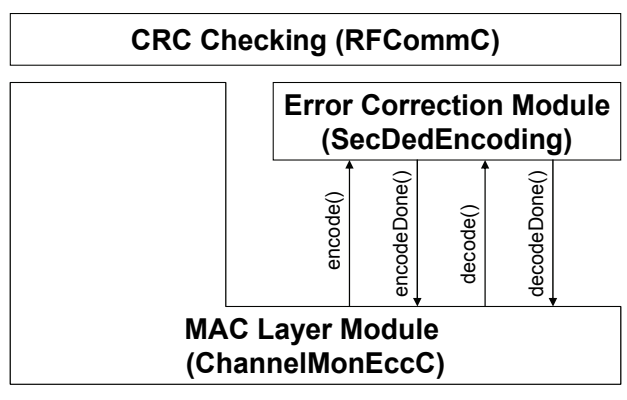

Radio Chip Module (SpiByteFifoC, ChipconC)

(b) Implementing error correction code with network stack

\begin{tabular}{|c|c|}
\hline $\begin{array}{l}\text { Preamble \& } \\
\text { Start Symbol }\end{array}$ & $\mathbf{n}_{\text {preay }}$ \\
\hline & $\begin{array}{c}\text { Payload \& } \\
\text { Checksum }\end{array}$ \\
\hline Encoded with ECC
\end{tabular}

(c) Packet length with error correction code

Figure 4. Providing error-correction code. 
The receiver can decode the corrected codeword by solving the equation $\mathbf{v}=\mathbf{u G}$. Especially, for a systematic code where the first $k$-columns of generator matrix $\mathbf{G}$ form an identity matrix, $\mathrm{u}$ is just first $k$-bits of $\mathbf{v}$.

2) Odd-weight-column code: Odd-weight column code can correct single bit errors and detect double bit errors (SECDED) [5]. As the name implies, each column of the parity matrix $\mathbf{H}$ has an odd number of $1 \mathrm{~s}$. To construct an odd-weight column code for an input of $k$ data bits, the parity matrix $\mathbf{H}$ should include a sufficient number of parity bits $r$ so that the number of columns of $\mathbf{H}$ is at least $k+r$. For example, $r=5$ parity bits are needed to recover $k=8$ bits of data, and $r=6$ parity bits are needed to recover $k=24$ bits of data.

The columns of $\mathbf{H}$ are constructed as follows:

- The last $r$ columns of $\mathbf{H}$ form the identity matrix $\mathbf{I}_{r}$.

- The first $k$ columns of $\mathbf{H}$ are chosen from any other odd weight column vectors than the ones used in $\mathbf{I}_{r}$.

For example, $\mathbf{G}, \mathbf{H}$ for $k=8, r=5$ are:

$$
\mathbf{G}=\left[\mathbf{I}_{\mathbf{8}}: \mathbf{C}\right]=\left[\begin{array}{lllllllllllll}
1 & 0 & 0 & 0 & 0 & 0 & 0 & 0 & 0 & 0 & 1 & 1 & 1 \\
0 & 1 & 0 & 0 & 0 & 0 & 0 & 0 & 0 & 1 & 0 & 1 & 1 \\
0 & 0 & 1 & 0 & 0 & 0 & 0 & 0 & 1 & 0 & 1 & 0 & 1 \\
0 & 0 & 0 & 1 & 0 & 0 & 0 & 0 & 1 & 0 & 1 & 1 & 0 \\
0 & 0 & 0 & 0 & 1 & 0 & 0 & 0 & 1 & 1 & 0 & 0 & 1 \\
0 & 0 & 0 & 0 & 0 & 1 & 0 & 0 & 1 & 1 & 0 & 1 & 0 \\
0 & 0 & 0 & 0 & 0 & 0 & 1 & 0 & 1 & 1 & 1 & 0 & 0 \\
0 & 0 & 0 & 0 & 0 & 0 & 0 & 1 & 1 & 1 & 1 & 1 & 1
\end{array}\right]
$$

We now give an example of how data is encoded, and how the received message can be corrected. Let the message being sent be $\mathbf{u}=\left[\begin{array}{lll}0100 & 0010\end{array}\right]$. The encoded message $\mathbf{v}$ is therefore

$$
\mathbf{v}=\mathbf{u} \mathbf{G}=\left[\begin{array}{lll}
0100 & 0010 & 10111
\end{array}\right]
$$

Assume that the second bit of the encoded message is inverted due to noise in the wireless channel. Then, received message $\mathbf{v}^{\prime}$ is [0100 0010 10111]. Multiplying the received message by the transpose of the parity matrix $\mathbf{H}$, we get the syndrome $\mathbf{s}$ as follows:

$$
\mathbf{s}=\mathbf{v}^{\prime} \mathbf{H}^{T}=\left[\begin{array}{lllll}
0 & 1 & 0 & 1 & 1
\end{array}\right]
$$

We note that the syndrome obtained is the second row of $\mathbf{H}^{T}$, which implies that second bit of the codeword is inverted. Thus, we can get correct codeword $\mathbf{v}=\left[\begin{array}{lll}0100 & 0010 & 10111\end{array}\right]$. Since the generator matrix $\mathbf{G}$ is a systematic code, the data bits can be decoded by taking the first- $k$ bits of the codeword. Thus, $\mathbf{u}=\left[\begin{array}{lll}0100 & 0010\end{array}\right]$.

3) Implementation: A wireless sensor node communicates with other sensor nodes using the network stack shown in Figure 1. Although error correction code (ECC) can be located in any other layer, we decided to have the error-correction- code module in the MAC layer, which interfaces application / network layer with physical layer by packetizing the received data bytes and fragmenting a packet to be sent into data bytes. Since this approach does not change the interface to the application/network layer, it has an advantage that any applications written for non-ECC version of MAC can run without any code modification. We implemented an error correction code module for odd-weight-column code SecDedEncoding, which takes 8-bit data and generates a 13-bit codeword.

Figure 4(b) shows how our error-correction-code implementation interacts with the MAC layer through the interface RadioEncoding. When a packet is to be sent, the MAC layer module ChannelMonEccC calls the method encode for each byte of data in the packet. After a sufficient number of input data bytes have been received, the input data bytes are encoded by the internal encoding function radio encode thread and codeword is passed to the ChannelMonEccC through encodeDone event. When data bytes are passed by the physical layer, the MAC layer module ChannelMonEccC calls the method decode for each byte of its received packet. After a sufficient number of input data bytes have been received, the received data bytes are decoded by the internal decoding function radio_decode_thread and the original data bytes are sent to $\bar{C}$ hannelMonEccC through 
decodeDone event. The internal encoding function radio_encode_thread calculates parity bits by comparing each bit of input data bytes. This corresponds to calculating $\mathbf{~} \mathbf{G}$, where $\mathbf{m}$ is the message and $\mathbf{G}$ is the generator matrix.

The internal decoding function radio_decode thread calculates the syndrome $\mathbf{s}$ by looking at each bit of received data bits. This is equivalent to $\mathbf{r} \mathbf{H}^{T}$ where $\mathbf{r}$ is received data and $\mathbf{H}^{T}$ is the transpose of the parity matrix. A non-zero syndrome implies an error and the position of bit errors can be found by comparing the syndrome with column vectors of $\mathbf{H}$ matrix. We made this lookup fast by using an array that maps any possible syndrome value to the error bit position.

In general, the MAC layer of WSN consists of the following fields: preamble, start symbol, payload and checksum (Figure 4(c)). When error-correction code is used, the data bytes in the payload and checksum are encoded into codeword while the data bytes for the preamble and start symbol are not encoded. Then, we can estimate the transmission overhead of an error-correction code for the following parameters:

- $n_{p r e}$ : length of preamble and start symbol (bytes)

- $n_{p a y}$ : length of payload and checksum (bytes)

- $r_{e c c}$ : length of codeword for one-byte data

- $n_{e c c}$ : data bytes transmitted with ECC (bytes)

- $n_{n o \_c c}$ : data bytes transmitted without ECC (bytes)

$$
\begin{aligned}
\text { Overhead } & =\frac{n_{e c c}-n_{n o_{-} e c c}}{n_{n o_{-} e c c}} \\
& =\frac{\left(n_{p r e}+n_{p a y} \cdot r_{e c c}\right)-\left(n_{p r e}+n_{p a y}\right)}{n_{p r e}+n_{p a y}} \\
& =\frac{n_{p a y}}{n_{p r e}+n_{p a y}} \cdot\left(r_{e c c}-1\right)
\end{aligned}
$$

Since the TinyOS distribution has $n_{p r e}=20$ and $n_{p a y}=$ 36 for CC1000 radio, the overhead for our ECC implementation can be calculated as follows:

$$
\begin{aligned}
\text { Overhead } & =\frac{n_{p a y}}{n_{p r e}+n_{p a y}} \cdot\left(r_{e c c}-1\right) \\
& =\frac{36}{20+36} \cdot\left(\frac{2 B}{1 B}-1\right)=64.3 \%
\end{aligned}
$$

\subsection{Reliable Transport Layer}

We wanted reliable communications. But we also wanted compatibility and ease of use. So we designed it to give it the same interface as that of existing best-effort transport layer. As shown in Figure 5(a), we designed the reliable transport layer so that it can be inserted between a best-effort transport layer and application layer without any significant modification to the application.

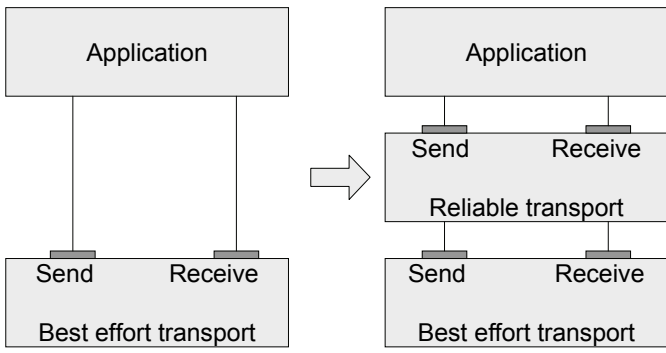

(a) Compatible interface of reliable transport layer

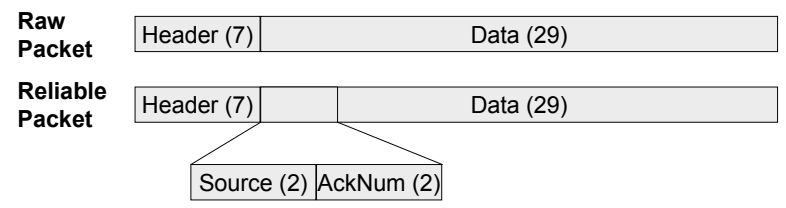

(b) Packet structure for reliable transport layer

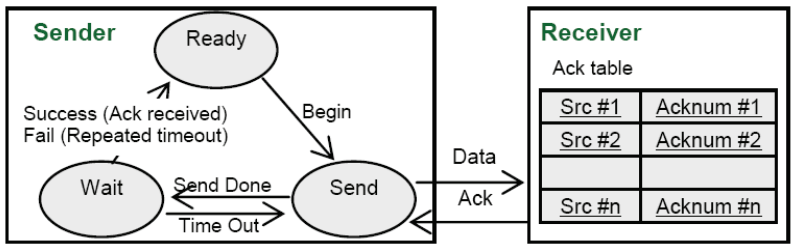

(c) Schematic of reliable transport

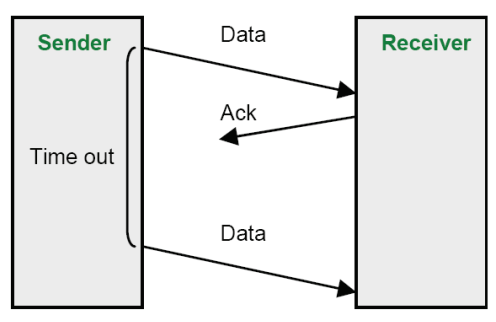

(d) Lost acknowledgement

Figure 5. Reliable transport layer.

We also wanted a lightweight layer. A compatible and lightweight approach steered us to implement connection-less communication. Interfaces of existing best-effort transport layer support only connection-less communication. In the reliable transport layer, Connection information is managed globally.

To guarantee reliable communication, we mainly used acknowledgment and retransmission. Packet structure came to incorporate more information. Sender and receiver use this information and react properly according to it.

1) Reliable message: For reliable communication, additional meta-data (source address, acknowledgment number) needs to be included in each packet. The packet size is 36 bytes. 7 bytes are already used by lower layers for meta-data. And 29 bytes are used as data field. 4 more bytes ( 2 for source address, 2 for acknowledgment) are taken from the data field for meta-data in the reliable 
transport layer. Now the length of the data field has decreased from 29 bytes to 25 bytes ( 13.8 percent loss).

Sender gets a packet from an upper application layer. And it realigns data, adds source address and acknowledgment number, and sends it to lower best-effort transport layer. Receiver also does the same conversion. The packet structure is shown in Figure 5(b). The use of additional meta-data is transparent to applications except the decreased size of the data field.

2) Sender: Sender is a finite state machine. When sender gets a packet from an upper application layer, it adds a source address and acknowledgment number as shown in the Figure 5(b), realigns data, and passes the packet to the lower best-effort transport layer. If the sender receives an acknowledgment from the receiver, it reports a success to the upper application layer. If it does not receive an acknowledgment but times out, it retransmits the unacknowledged packet. The amount of waiting time is a random number between $\mathrm{T}$ and $2 \mathrm{~T}$. After $\mathrm{N}$ successive time-outs, the sender reports a failure to the upper application layer. For simplicity, the sender uses block-and-wait strategy. The sender only needs to remember the current receiver's information. Figure 5(c) shows the main part of a state diagram of the sender. Since there is no queue in the lower layer, if sender tries to send a packet while the receiver of the same node is also replying by sending an acknowledgment, the packet from the sender can be lost. So a buffer of size 1 is used by the sender. When an acknowledgment is being processed, sender saves the packet in the buffer and transmits after the receiver of that sensor completes the acknowledgment.

3) Receiver: Receiver is also a finite state machine. When the receiver gets a packet from the lower best-effort transport layer, it looks at the source address and acknowledgment number. If it is a new packet, it sends an acknowledgment and passes the packet to the upper application layer. If it is a packet already received, it only sends an acknowledgment to the sender. To decide whether the received packet is a new packet or an already received one, it maintains connection information in the Ack table. The table has a pair of source addresses and acknowledgment number as an entry. In case of a lost acknowledgment, as in Figure 5(d), duplicate data packets can arrive. Then we should not report the second packet, and we need a table for this purpose.

Since the size of table is limited, it cannot handle an arbitrary number of connections all the time. So a FIFO algorithm is used to replace entries in the table. To reduce table lookup time, a reverse chronological search is used. It looks up the most recent connection first, and then next recent connection, and so on.

\section{Evaluation}

In order to see the effectiveness of our network stack implementation, we set up two kinds of experiments: range test and contention test. The range test, which was set up in the middle of the UC Berkeley campus (Figure 6 ), is to measure the performance of the network stack in a non-contentious environment. In the range test, the sender sends a number of packets and the receiver counts how many packets it received from the sender as we moved the sender farther from the receiver. The contention test, which was set up in an indoor environment, is to measure the performance of the network stack in the presence of traffic as we vary the number of senders or the number of radio channels being used. For each test, we used the packet reception rate as an indicator of effectiveness for the transmission method.

\subsection{Range Test}

In order to see whether our network stack performs reasonably in a non-contentious environment, we measured the packet reception rate as we vary the distance between the sender and the receiver. As a preliminary test, we measured the performance only with the basic configuration of the network stack without using any additional functionality such as error-correction code, retransmission or multiple radio channels. Figure 7(a) shows that the network stack performs well up to $800 \mathrm{ft}$ with the packet reception rate higher than $90 \%$. But, the packet reception rate drops severely after this point.

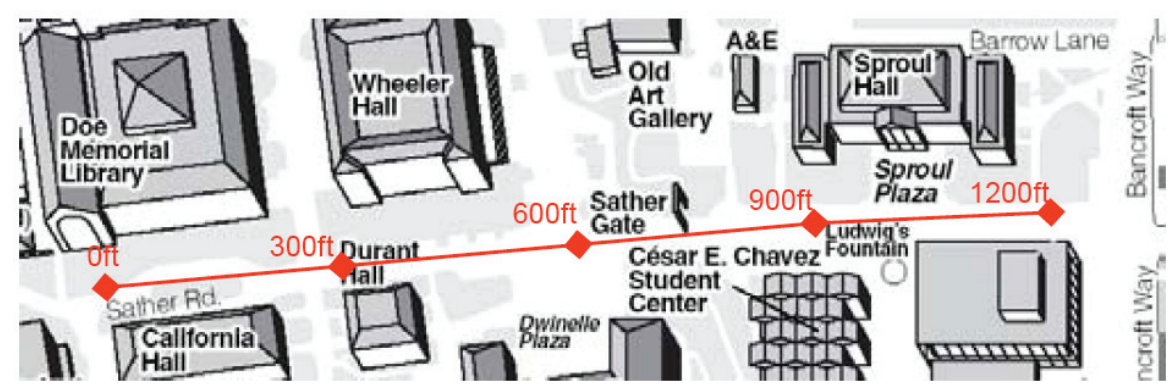

Figure 6. Locations of outdoor experiment. 


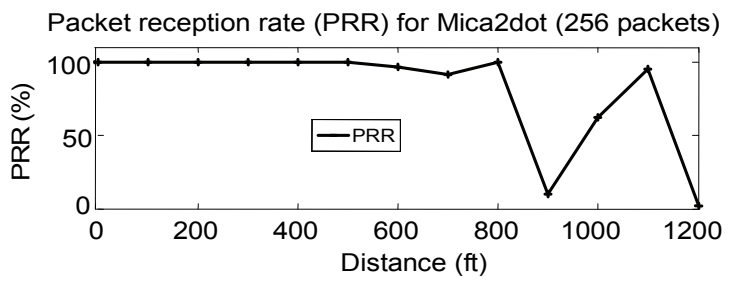

(a) Base-case

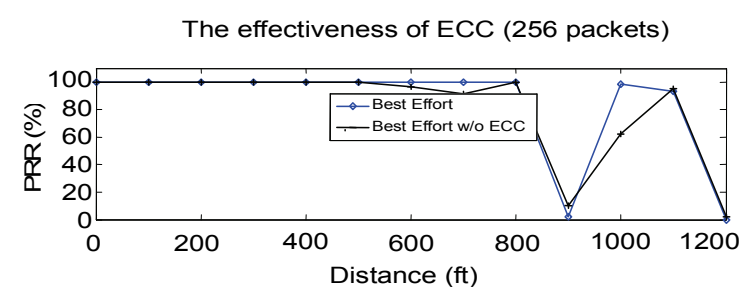

(b) Effectiveness of ECC

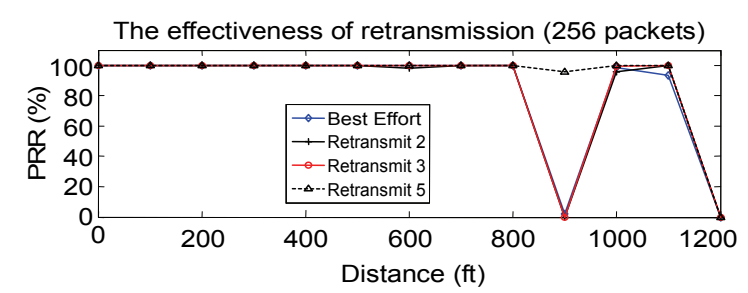

(c) Effectiveness of retransmission

Figure 7. Range test.

In order to see whether additional functionality can improve the performance, we first tested the case with error-correction code. We measured the performance of the network stack with SECDED error-correction code and compared it with the performance with the base case.
Figure 7(b) shows that the ECC implementation was more resilient to errors and had a better packet reception rate. However, the SECDED code is not effective when the packets were completely lost $(900 \mathrm{ft})$.

We have seen that using SECDED error correction improves the performance of the base case, but it still has a problem of packet losses. In the next test, we used both error-correction code and retransmission to see whether we can further improve the performance of the network stack. We measured the packet reception rate of the network stack for the four different implementations: the implementation with no retransmission and the ones with 2,3 or 5 retransmissions (Figure 7(c)). All implementations used SECDED for integrity. Retransmission was slightly better than the best effort transmission. The difference between the three retransmission schemes were not that noticeable except that 5 retransmission could receive the message while all the other methods failed at $900 \mathrm{ft}$. We found this was possible because radio waves from the sender took different paths when the sender retransmitted the packets.

\subsection{Contention Test}

In order to see how effective retransmission handles contentions, we set up an experiment. We placed multiple senders $(1,2$ or 4$)$ and a receiver close by and measured the packet reception rate and the transmission time for two extreme cases: no retransmission and 5 retransmissions. Figure 8(a) shows that the effect of retransmission in a closely populated area is very noticeable.
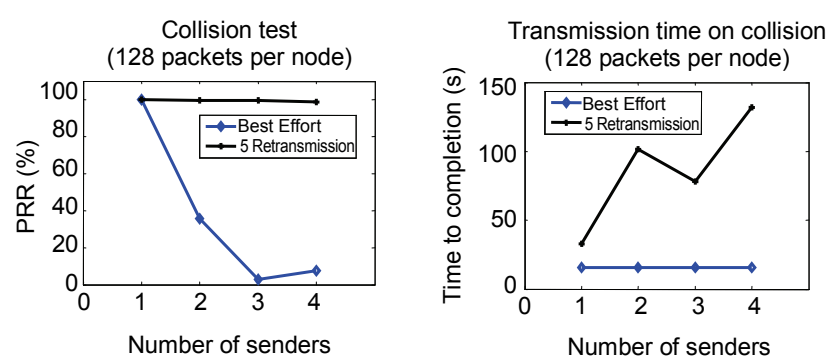

(a) Effect of retransmission
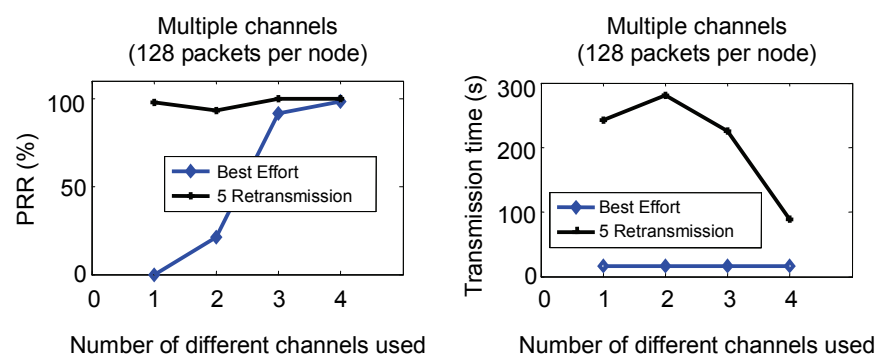

(b) Effect of using multiple channels

Figure 8. Contention test. 
Table 4. Time to send / receive 512 packets.

\begin{tabular}{ccc}
\hline Best Effort & $\begin{array}{c}\text { Retransmission } \\
\text { (5 retries) }\end{array}$ & $\begin{array}{c}\text { Retransmission } \\
\text { (0 retries) }\end{array}$ \\
\hline $31 \mathrm{sec}$ & $64 \mathrm{sec}$ & $32 \mathrm{sec}$ \\
\hline
\end{tabular}

It reduced most of the packet drops due to collision with increased transmission time. This shows that packets are very likely to be dropped when multiple nodes are sending packets in bursts and that packet drops can be avoided with retransmission. We can also see that retransmission takes more time as the rate of collision increases.

In the next experiment, we wanted to see the effect of multiple radio channels. We prepared 8 nodes, dividing them into groups, each of which is composed of one sender and one receiver. We measured the packet reception rates and the transmission time for the non-retransmission implementation and the 5 retransmission implementation of the four senders. We varied the number channels used (1,2 and 4) to see the effect of multiple channels on the two implementations. Figure 8(b) shows that using multiple channels was more effective with the non-retransmission implementation than retransmission implementation. This was expected from the results of the range test in that retransmission received most of the packets whereas non-retransmission received only $10 \%$ of the packets. Using multiple channels also helped the retransmission time. It used smaller amounts of transmission time when more channels are available. When there are fewer channels available, it spent more for transmission but it still achieved a high packet reception rate. This implies that retransmission and the use of multiple channels can be beneficial for reliable packet delivery. We can also infer that there is some interference among channels. Otherwise, for the case of 4 channels, the ratio of received packets should be very close to $100 \%$ for the best effort transport.

\subsection{Overhead of Reliable Transport Layer}

To measure the overhead of the sender, we eliminated the wait for acknowledgment on the sender side. And for 512 packets, we measured completion time. The result is shown in Table 4. The overhead is negligible for the sender. To measure the overhead of the receiver, we made the receiver send data to another sensor node. However, the two sensor nodes interfered with each other. Unfortunately we were not able to get correct results. We surely expect some overhead for the receiver side, because it should send a packet for each incoming packet while this is not needed in the best effort transport. In retransmission, every packet involves two transmissions. This explains why retransmission takes about

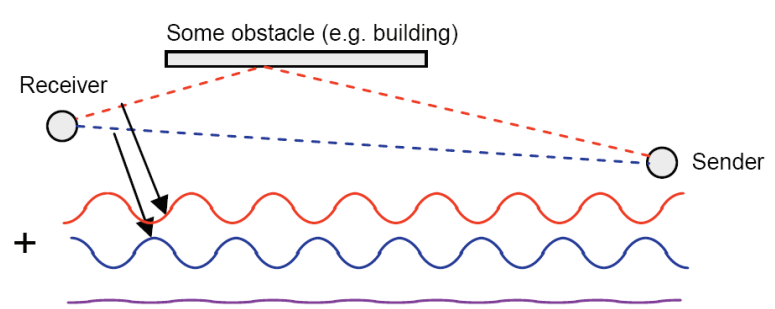

Figure 9. Multi-path effect.

twice as long as the best effort does.

\subsection{Multi-Path Effect and Theoretical Limit of Range}

If we look at the range test results in the previous Figures, the graphs consistently had dips at $900 \mathrm{ft}$. Once the sender moves beyond that distance, the receiver received the packets from the sender again. This happened because the radio signal is propagated through waves. Radio waves from the sender take paths while they travel and their phase can change when they reflect on some obstacles. Waves of opposite phase cancel each other out and the resulting signal becomes weaker than the sensitivity of the receiving node, thus packets cannot be heard. This phenomenon is called multi-path effect (Figure 9). More complicated devices like CDMA cellular phones use multiple antennas of different phase to avoid the antenna of different phase to avoid problem, but we cannot depend on this method because $\mathrm{CC} 1000$ has only single antenna. However, we can around this by having intermediate nodes between the two nodes and by having the intermediate nodes relay the packets.

\section{Conclusions}

We presented an implementation of the network stack for the $\mathrm{CC} 1000$ radio transceiver. It has reasonable performance in an outdoor environment with the packet reception rate close to $100 \%$ up to $800 \mathrm{ft}$. To improve its performance in a more contentious environment, we extended the network stack with error-correction code, reliable transmission and multiple radio channels. For error-correction code, we used a SECDED code, and it was effective in improving the packet reception rate except when the packets were completely lost due to the multi-path effect. The reliable transmission scheme was effective in reducing packet loss from the multi-path effect and contention from traffic, but its overhead was a bit high when there was a great deal of collision. This was caused by some of the implementation decisions. In our reliable transmission scheme, senders try to retransmit unacknowledged packets after a random amount of time within the timing window of fixed size. We found 
that this does not help under high collision even though the waiting time varied within the timing window. We expect that increasing the timing window size similar to exponential back-off will reduce the transmission rate so that the overall system can make progress. In the reliable transport layer, the sender's window size is one and this causes the sender to block and wait. Increasing the window size will reduce the waiting time and improve the transfer rate. This requires that the sender keeps an 'Ack table' to buffer unacknowledged packets. Using multiple radio channels was very effective in reducing collisions when multiple senders burst packets. Currently, the channel is statically tuned at compile time, but performance can suffer when the channel is mis-configured or there is contending traffic at the configured channel. A dynamic frequency allocation mechanism is needed to address this problem.

\section{Acknowledgements}

This work is supported by the Defense Advanced Research Projects Agency under a contract F33615-01C1895 ("NEST"), the National Science Foundation under grants \#0435454 ("NeTS-NR") and \#0454432 ("CNS-CRI"), a grant from the Keck Foundation, and generous gifts from HP and Intel.

\section{References}

[1] K. Sohrabi, B. Manriquez, and G. J. Pottie, "Near ground wideband channel measurement in 800 - 1000mhz," IEEE Vehicular Technology Conference, July 1999.

[2] A. Woo and D. E. Culler, "A transmission control scheme for media access in sensor networks," In The Annual International Conference on Mobile Computing and Networking (MobiCom'01), July 2001.

[3] J. Zhao and R. Govindan, "Understanding packet delivery performance in dense wireless sensor networks," In The ACM Conference on Embedded Networked Sensor Systems (SenSys'03), November 2003.

[4] Cc1000 data sheet. http://www.chipcon.com/files/CC1000 Data_Sheet_2_1.pdf.

[5] M. Y. Hsiao, "A class of optimal minimum odd-weightcolumnsec-dedcodes," IBM Journal of Research and Development, Vol. 14, No. 4, July 1970.

[6] J. Jeong and C.-T. Ee, "Forward error correction in sensor networks," In The First International Workshop on Wireless Sensor Networks (WWSN'07), June 2007.

[7] J. Hill. Cc1000 network stack. http://local.cs.berkeley. edu/grad/jaein/xbow.tgz.

[8] Mica2dot. http://www.xbow.com/products/Product_pdf files/Wireless_pdf/MICA2DOT_Datasheet.pdf.

[9] Atmega 1031 microprocessor data sheet. http://www. atmel.com/dyn/ resources/prod_documents/doc0945.pdf. 